\title{
The timing of interresponse intervals*
}

\author{
ALAN M. WING and A. B. KRISTOFFERSON \\ McMaster University, Hamilton, Ontario, Canada
}

\begin{abstract}
Analogs of models of duration discrimination are here related to the timing of discrete motor responses. The measure of interest is the variability in duration of intervals collected in short interval reproduction tasks. For data from a Morse key-tapping task, it is shown that, taken separately, neither of the models described can completely account for the relation between the mean and the variance of the interresponse intervals.
\end{abstract}

Michon (1967) states that explanations to account for temporal aspects of behavior are similar for time perception and for timing of discrete responses and rhythmic performance. If it were the case that response timing (as manifest in some simple skill such as the periodic tapping of a Morse key) and time perception do reflect the operation of one and the same duration mechanism, then recent theoretical accounts of duration discrimination (Creelman, 1962; Allan, Kristofferson, \& Wiens, 1971) might have application to response timing.

As a theoretical account of a timekeeping mechanism underlying the timing by a $S$ of an interval between two successive responses, consider the following stochastic wait process. After $\mathrm{S}$ has made his first response, suppose he withholds his next response until a given number, $n$, of hypothetical, internal events has occurred, where the delays between successive internal events are described by a random variable, $y$, with probability density function, $f(y)$. The resulting interresponse interval (I) will be a waiting time given by the sum of the $\mathrm{n}$ event delays (assuming the first response coincides with the zeroth event). Provided successive interevent delays are independent, the distribution of waiting times, $f_{n}(I)$, is given by the convolution,

$$
\sum \mathrm{f}(\mathrm{y})
$$

of the $n$ event delay distributions, so that an increase in $\mathrm{n}$ results in both larger mean, $\mu_{\mathrm{I}}$, and variance, $\sigma_{\mathrm{I}}^{2}$, of waiting times. Thus, if the Is are drawn at random from $f_{n}(I)$, for longer intervals (achieved by increasing $n$ ), a linear increasing relation with zero intercept should hold between the observed mean and variance.

This model is essentially an extension of the account of two-alternative forced-choice duration discrimination by Creelman (1962). The experimental procedure presents two intervals of duration $T,(T+\Delta T)$ on each trial in random order, and S's task is to state whether a

*This research was performed under Grant No. A7919 from the National Research Council of Canada. prespecified interval was in first or second position on a given trial. Creelman assumed a Poisson source of internal events and suggested that a count is taken of the number of these events which occur during each interval to be judged. This is a random variable, and S's decision problem is viewed as the determination of the likelihood ratio of an observed count of events under the count distributions corresponding to the two alternative intervals. Two intervals which differ in duration by a fixed amount, $\Delta \mathrm{T}$, will result, on average, in the same difference in internal count, whatever their base duration, T. However, with larger counts, deriving from longer base durations, there is associated greater variability. Thus, it is a prediction of Creelman's model that performance is inversely related to base duration. For auditory stimuli in some situations this prediction is confirmed.

Allan, Kristofferson, and Wiens (1971) have obtained data for duration discrimination of brief light flashes, which supports a prediction of no effect of base duration on performance derived from another model of the temporal mechanism. They assume that errors in duration discrimination are the result of variable afferent delays in the registration of the neural signals marking interval onset and offset and that the timing mechanism contributes no variability to the measure on which the decision of long or short interval is based. Assuming the distribution of afferent delays is not a function of interval duration, the decision statistic should depend only on differences in duration between two alternative intervals and not on their base duration.

As response timing analog to the afferent delay model, we consider a model closely related to that of McGill (1962), in which a deterministic timekeeper triggers responses, each of which is subject to a random efferent delay, $D_{j}$. The interresponse interval is then given by $\mathrm{I}=\mathrm{C}-\mathrm{D}_{1}{ }^{+}+\mathrm{D}_{2}$, where $\mathrm{C}$ is the constant period of the timekeeper and, if successive efferent delays are independent and have variance $\sigma_{D}^{2}, \sigma_{I}^{2}=2 \sigma_{D}^{2}$. Thus, provided $\sigma_{\mathrm{D}}^{2}$ does not depend on the period, $\sigma_{\mathrm{I}}^{2}$ will remain constant over changes in $\mu_{\mathrm{I}}$.

In a procedure used by Treisman (1963), a time 


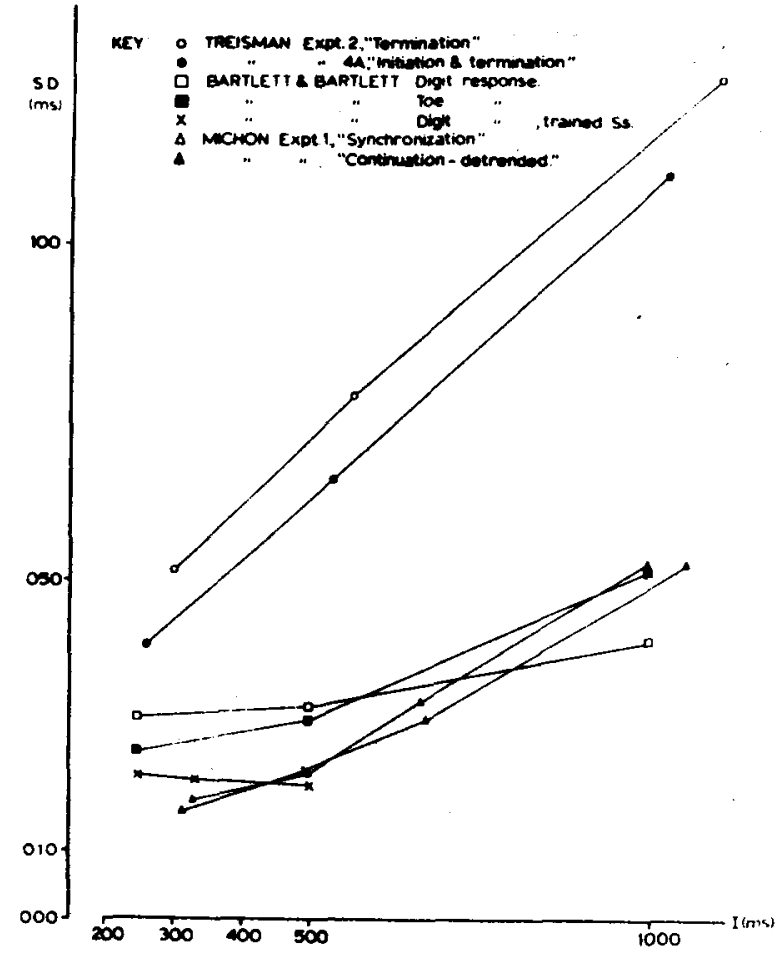

Fig. 1. Standard deviation of reproduced interval averaged over Ss as a function of mean interval. Data replotted from Treisman (1963; Tables 2 and 4), Michon (1967; Table 2), and Bartlett \& Bartlett (1959; Tables 2 and 3).

interval was presented on each trial as the duration of a continuous auditory stimulus. In his Experiment 2, following a constant, 2 -sec warning interval, the tone came on for a preselected duration $(\mathrm{T})$ in the range of $250-3,000 \mathrm{msec}$. After a randomly selected, silent interval in the range of $500-2,500 \mathrm{msec}$, the tone came on again. $S$ was then required to terminate it by depressing a reaction key so that the two tones would appear to be of equal duration. In his Experiment 4A, the procedure was the same except that $S$ had to tap the reaction key to initiate as well as to terminate the second tone.

Within each block of 10 trials, the same value of $T$ was used. The design of Experiment 2 required Ss to estimate durations with and without presentation of $\mathrm{T}$ on alternate blocks; Treisman referred to these as methods of reproduction and production, respectively. For the four Ss in each of the four sessions, all values of $\mathrm{T}(250,500,1,000$, and $3,000 \mathrm{msec})$ occurred. Each value was used for both the methods before the next $T$ was selected. In Experiment $4 \mathrm{~A}$, the design was changed to run only two sessions, with the two methods in separate sessions counterbalanced over Ss. The value of $\mathrm{T}$ for each block was selected in a regular ascending-descending fashion in both Experiments 2 and 4A. The reproduction results are redrawn in Fig. 1 for the two experiments. Plotting the averages over Ss of mean reproduced interval against the corresponding estimates of the standard deviations $\left(S_{I}\right)$, the shape of the functions is a straight line of slope close to unity.

A procedure used by Michon (1967) presented intervals as the time between very short, loud auditory clicks, and Is were defined by successive taps of a telegraph key. Two sequences of 200 intervals each were taken at each of six Ts in the range of $333-3,333 \mathrm{msec}$ under two experimental conditions, "continuation" and "synchronization." Synchronization required Ss to tap in phase with $\mathrm{T}$ for all 200 intervals. In the continuation mode, Ss were given just 10 advance stimulus intervals, with which they usually synchronized within three or four clicks. Then, without interruption, they made the required 200 Is without the clicks. Michon's data averaged over Ss is replotted on the same axes as Treisman's data (Fig. 1). There appears to be little difference in the two functions for continuation and synchronization, each being "somewhat curvilinear with increasing slope. Considerably reduced $\mathrm{S}_{\mathrm{I}}$ is evidenced in these sequential data compared with the data from Treisman.

Bartlett and Bartlett (1959) required Ss to attend to a sequence of Ts and to strike a response key to coincide with one of the train of auditory clicks when they were satisfied that they were "following the rhythm." The S's response terminated the sequence, and the subsequent $T$ value was selected from a range of $125-4,000 \mathrm{msec}$ in one and a range of $167.500 \mathrm{msec}$ in the other of the last two experiments reported. In the former, Ss used finger or toe responses and the $\bar{I}$ vs $S_{I}$ functions deviate from linearity in being concave upward, as can be seen from the averaged data in Fig. 1. However, in this experiment, two Ss with proven timing skills showed no increase in $S_{I}$ as $\bar{I}$ increased.

Thus, the experiments as summarized in Fig. 1 do not generally support the prediction of a linear increasing relation between $\mu_{\mathrm{I}}$ and $\sigma_{\mathrm{I}}^{2}$. In fact, in all cases, except one, $S_{I}^{2}$ increases as an accelerating function of $\bar{I}$, so that both proposed models appear to fail. The only result consistent with either model was obtained with the "expert" Ss of Bartlett and Bartlett in a task where the interval to be timed was presented repeatedly before $S$ was required to produce it. For this finding, the more appropriate model involves the deterministic timekeeper such that the interval is given by $\mathrm{I}=\mathrm{A}+\mathrm{C}+\mathrm{D}$, where $\mathrm{A}$ is a random variable representing the afferent delay, since Bartlett and Bartlett's Ss were in effect terminating a stimulus-initiated interval.

In this paper, we describe experimental results for highly trained Ss employed in a paradigm similar to Michon's continuation task, but with the value of $T$ held constant over several successive sessions. Since we were reluctant to assume that the functional relation between $\mu_{\mathrm{I}}$ and $\sigma_{\mathrm{I}}^{2}$ is continuous, the spacing of the $T$ values is much closer than in the earlier studies reviewed. We have chosen to examine the function for response 
Fig. 2. Experimental event sequence.
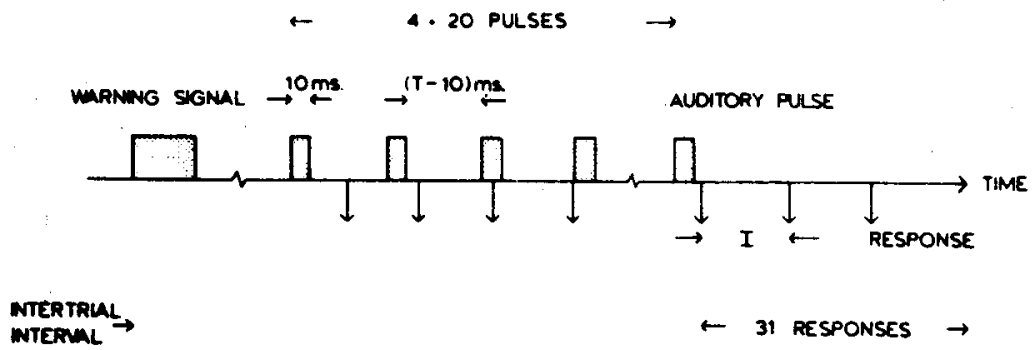

WTERM

$\leftarrow 31$ RESPONSES $\rightarrow$

숭.

rates close to the upper limit for the particular response selected.

\section{METHOD}

The task required of $S$ was a combination of paced and unpaced tapping of a telegraph key. Following a warning signal (see Fig. 2), a sequence of $10-\mathrm{msec}$-duration $2,000-\mathrm{Hz}$ auditory pulses separated by intervals, $(T-10) \mathrm{msec}$, was presented binaurally. The pulses were clearly audible (sound level, $62 \mathrm{~dB}$ re $38 \mathrm{~dB}$ background) over headphones worn by $\mathrm{S}$.

$S$ was instructed to depress the key periodically to close the contact in synchrony with the pulse train; if a response led or lagged any given pulse onset by more than $35 \mathrm{msec}$, visual feedback was given immediately, indicating the direction of error. With practice, Ss had no difficulty staying within this time window. To assist synchronization, $S$ was not given feedback, nor was there recording of response times, on the first four pulses. After that, if a response was skipped, the sequence automatically aborted and began over again.

Following the last pulse of the train of 24 pulses in the synchronization phase, $\mathrm{S}$ was required to continue tapping at the established, fixed rate for a further 31 responses (the continuation phase). The warning signal was sounded on the last response in the continuation phase to indicate to $S$ that he should stop. An interval of approximately $13 \mathrm{sec}$ preceded the next warning signal and ensuing sequence.

The telegraph key was clamped in a fixed position relative to $S$ 's chair. A downward vertical movement of less than $0.3 \mathrm{~cm}$, requiring a weight of $120 \mathrm{~g}$, made electrical contact for recording purposes. The S's arm was in no way constrained, but he was encouraged to adopt a habitual mode of responding. The experiments took place with $\mathrm{S}$ seated in a sound-attenuated room. In another room, a PDP-8 computer was used to control stimulus event times and to record Is with an accuracy to the nearest $1 \mathrm{msec}$.

Values of $T$ ranged from 180 through $350 \mathrm{msec}$ in Experiment 1 and 170 through $270 \mathrm{msec}$ in Experiment 2, and a single value of $T$ was used for 10 sessions before another value was run. Order of assignment of $T$ conditions was pseudorandom in each experiment and different for each $\mathrm{S}$. Each session lasted about $35 \mathrm{~min}$ and consisted of five blocks of 11 sequences. Where possible, the last four sessions for each value of $T$ were run two per day on consecutive days. The earlier data were considered as practice runs. In the case of J.W. in Experiment 1, it was possible to run only four sessions in the practice groups, thus giving groups of only eight sessions in all at each T. In Experiment 2, all the Ss were run according to this latter schedule after a break of 3 months. Three paid Ss and the first author took part. All were right-handed.

\section{RESULTS}

The results presented in this paper are for the last 30 Is collected in the continuation phase of sequences run in the last four sessions under each value of $T$. Occasionally, Ss reported missing a response or making a double response. Such sequences, identifiable by intervals which are nearly a multiple of $T$, were eliminated from the analyses. Less than $5 \%$ of sequences under each $\mathrm{T}$ had to be rejected for any $\mathrm{S}$.

\section{Distribution of Is}

All four Ss yielded relative frequency distributions on I which were smooth, single-peaked, and symmetrical at every value of $T$. Four representative histograms are shown for M.F. in Fig. 3. Each comprises more than 6,000 observations. Given the obvious regularity of the distributions, it is appropriate to use the mean and variance as summary statistics.

\section{Stationarity of Is}

As an indicator of the presence of consistent

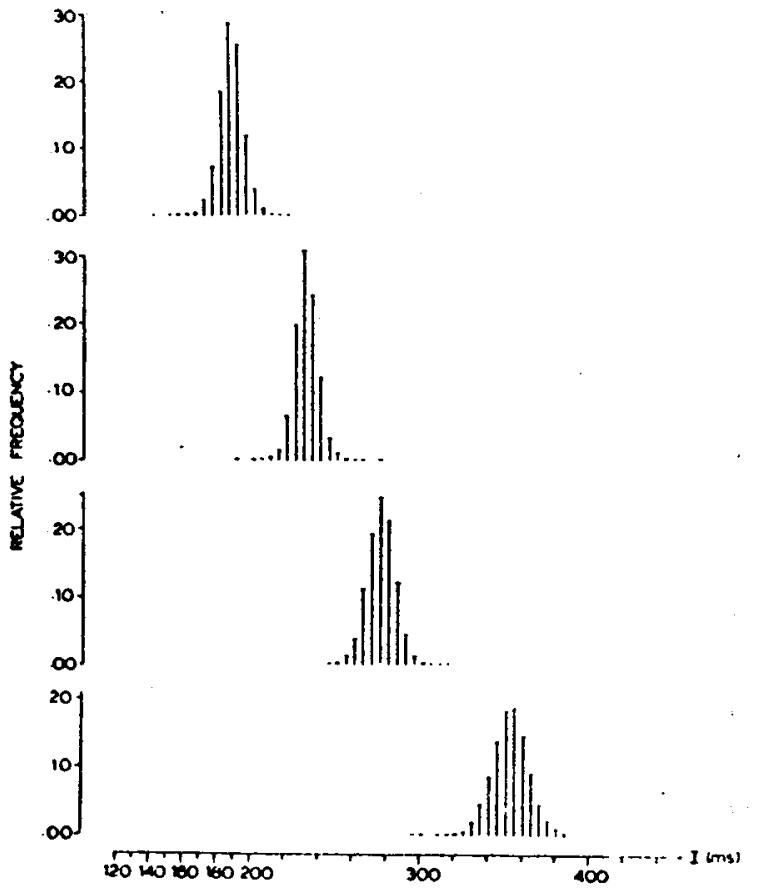

Fig. 3. I histograms for M.F. at standards of 180, 220. 270. and 350 msec. Class interval $=5 \mathrm{msec}$. 
Table 1

Average Increase in Mean from First to Second Half of Each Sequence for MF (in Milliseconds)

\begin{tabular}{lcccc}
\hline & \multicolumn{4}{c}{ Standard } \\
\cline { 2 - 5 } & 180 & 220 & 270 & 350 \\
\hline Change in $\overline{\mathrm{I}}$ & +1.23 & +2.10 & +0.17 & +0.54 \\
SD & 2.12 & 2.11 & 2.50 & 3.44 \\
N (Sequences) & 212 & 209 & 203 & 209 \\
\hline
\end{tabular}

Table 2

Average Increase in Mean from First to Second Half of Each Sequence Over All Standards in Experiments I and II (in Milliseconds)

\begin{tabular}{lrrrr}
\hline & \multicolumn{4}{c}{ Subject } \\
\cline { 2 - 5 } & AW & \multicolumn{1}{c}{ JW } & IT & MF \\
\hline Change in $\bar{I}$ & +0.69 & +1.36 & +0.33 & +0.81 \\
Pooled SD & 2.26 & 2.23 & 3.14 & 2.52 \\
\hline
\end{tabular}

monotonic increasing or decreasing trends in the sequence means, for each $S$ the estimates, $\bar{I}$, were calculated separately for first and second halves of each sequence, the second value subtracted from the first, and the mean and standard deviation of these differences calculated over all sequences at a given standard. Table 1 gives representative results for M.F.

In general, for each $\mathrm{S}$, there was no effect of $\mathrm{T}$ on the differences. The results, pooled over standards for each $S$, are presented in Table 2 . While there were consistent increases in the mean, these were so small on average as not to warrant a detrending transformation.

\section{Experiment 1}

The variance estimate $S_{I}^{2}$ was obtained by taking the mean of the individual sequence variances at each value of $T$. This was chosen in preference to calculating the mean squared deviation about the grand mean for all sequences at a given $T$ (overall variance), since the latter quantity may include variability in S's perceived value of $T$ as set up in synchronization. At this stage, we do not attempt an account of this dynamic aspect of temporal performance. Figure 4 shows $S_{I}^{2}$ as a function of $\bar{I}$ for individual $S$ data and the averages thereof (solid line). The experimental error in $S_{\mathfrak{I}}^{2}$ was quite consistent over Ss and is shown for M.F. On average, Ss slightly overestimate $T$ by about $2 \mathrm{msec}$, and, whereas $S_{I}^{2}$ increases with $\bar{I}$ above $250 \mathrm{msec}$, in the range of 180 through $250 \mathrm{msec} \mathrm{S}_{\mathrm{I}}^{2}$ is nearly constant.

\section{Experiment 2}

Figure 5 gives $S_{1}^{2}$ as a function of $\bar{I}$ for $T$ values of 170,220 , and $270 \mathrm{msec}$. The average level of $\mathrm{S}_{\mathrm{I}}^{2}$ is noticeably increased over that for Experiment 1 , especially for $T=170 \mathrm{msec}$. At this standard, A.W. and J.W. reported difficulty in physically causing their responses to happen. It will be noted that I.T., in overestimating $T$ on average by about $8 \mathrm{msec}$, was not responding at as fast a rate as the other $S$ s at $T=$ $170 \mathrm{msec}$, nor did he report any difficulty in responding.

\section{DISCUSSION}

The general level of $S_{I}^{2}$ in the experiments reported here was considerably less than for the continuation series of Michon (1967). This may be due, in part, to longer training of our Ss and the fact that they were required to reproduce only one value of $\mathrm{T}$ in any session. (Michon's primary interest was in the dynamics of synchronous temporal tracking behavior.) The possible sensitivity of the level of $S_{I}^{2}$ to practice lends a cautionary note to the selection of experimental procedures for the estimation of the $\mu_{I}$ vs $\sigma_{I}^{2}$ function until more is known about practice effects. In fact, from

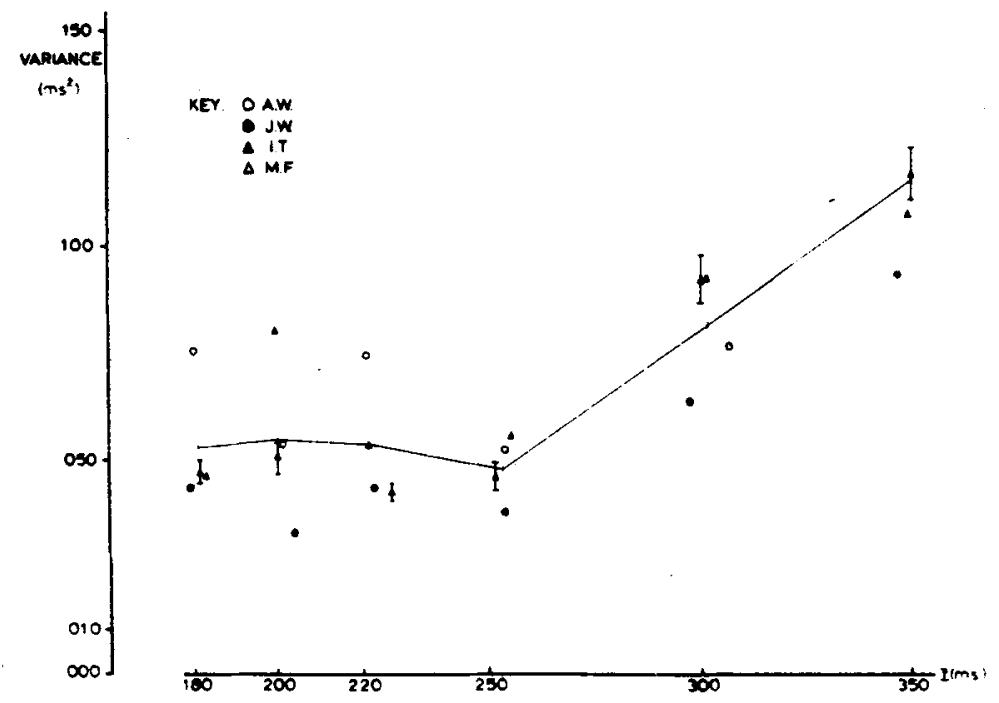

Fig. 4. Mean sequence variance, $S_{I}^{2}$, as a function of mean, $\bar{I}$ (Experiment 1 ). Two standard errors on the estimate $S_{I}^{2}$ shown for M.F. 
Fig. 5. $S_{I}^{2}$ as a function of $\bar{I}$ (Experiment 2). Two standard errors on the estimates $S_{\mathbf{I}}^{2}$ shown for M.F. Dashed curves indicate average functions for Experiment 1 .
Fig. 6. Schematic of two-stage response timing mechanism.
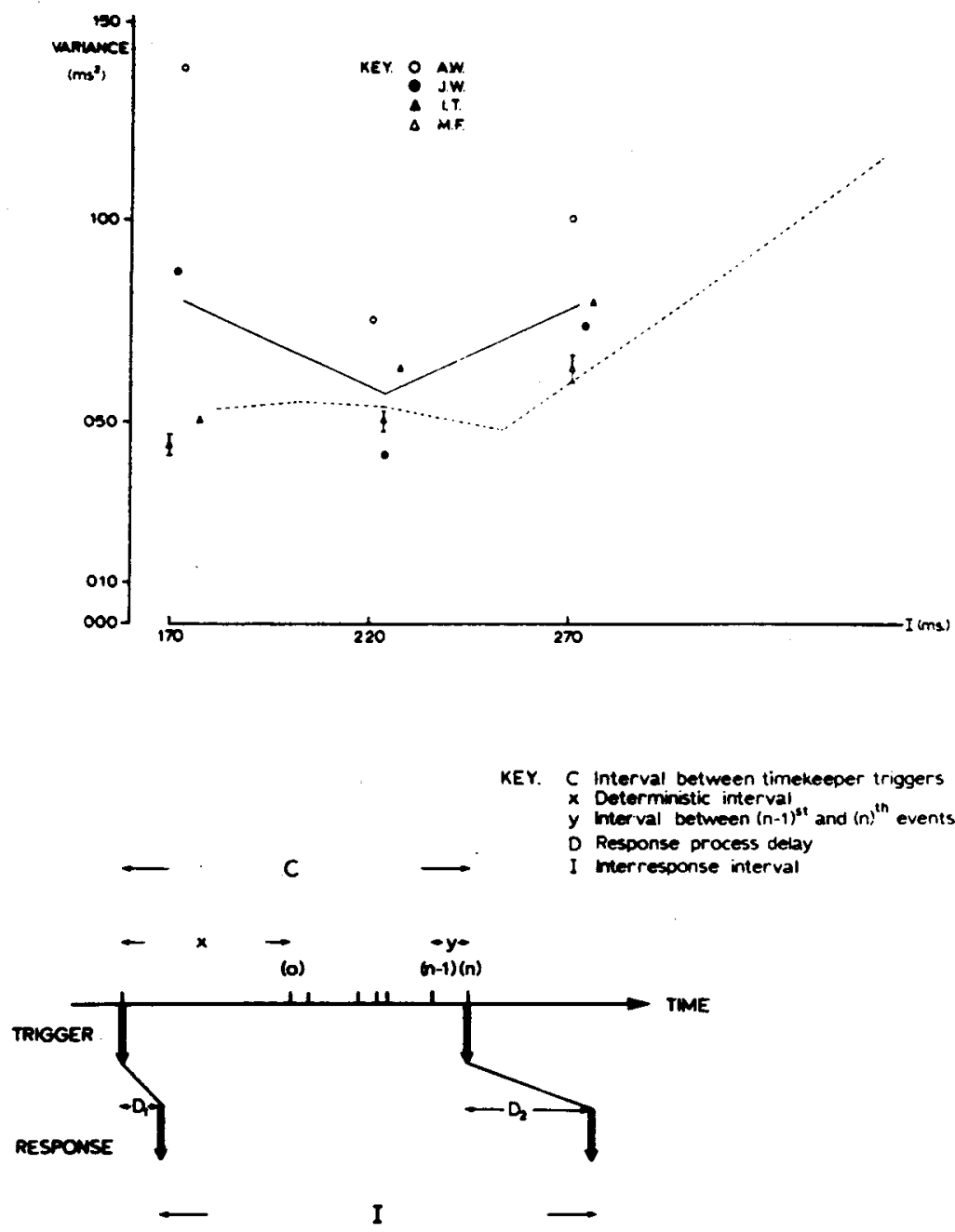

Experiment 1 to Experiment 2, there is a slight increase in mean level of $S_{I}^{2}$, which might be a result of fewer practice sessions in the latter case.

In Experiment 1, the functional relation between $S_{\mathbf{I}}^{2}$ and $\bar{I}$ comprises two nearly linear segments. Over the range of Is, 180 through $250 \mathrm{msec}, \mathrm{S}_{\mathrm{I}}^{2}$ is constant in value. This accords with Bartlett and Bartlett's (1959) result for trained Ss, although it will be noted that the variance in their study is greater. However, for Is in the range of 250 through $350 \mathrm{msec}, S_{I}^{2}$ increases with $\bar{I}$ in a linear fashion but with an (extrapolated) intercept on the $I$ axis for zero variance which is greater than zero. This picture, given by the overall average function, is also found for individual Ss, although significant, but apparently nonsystematic, discrepancies do occur. One is therefore led to the conclusion that the deterministic timekeeping mechanism with variable response delays is more appropriate for Is up to $250 \mathrm{msec}$, whereas above that value we should entertain the stochastic wait model with a modification to take into account the nonzero origin. The modification provides that a time interval of $250 \mathrm{msec}$ is produced by the deterministic timekeeper and that a stochastic wait is invoked to produce an additive interval to the fixed interval to make up longer intervals (see Fig. 6). For such a hybrid model, the resultant Is would exhibit a linear increasing relation between $\sigma_{I}^{2}$ and $\mu_{I}$ except for intervals of less than $250 \mathrm{msec}$, which would have constant variance, as for the basic deterministic model. That is, the additional variance of the response delay process would have to be taken as additive and constant over all values of $T$ if the form of the variance relation of the underlying timekeeping mechanism was to be estimated from $S_{I}^{2}$. Whether this assumption is reasonable over a large range of $T$ is not known. Certainly, at the shortest $T$ of $170 \mathrm{msec}$ in Experiment 2, the fact that Ss complained of difficulty in outputting their responses "to command" should lead us to question whether successive response delays will be independent and of the same variance as at the other response rates. The assumption that these delays can be described as samplings of a random variable with fixed variance may not be reasonable. but a direct means of testing this is not yet available. 


\section{REFERENCES}

Allan. L. G., Kristofferson. A. B., \& Wiens. E. IV. Duration discrimination of brief light flashes. Perception \& Psychophysics, 1971. 9, 327-334.

Bartlett. N. R., \& Bartlett. S. C. Synchronization of a motor response with an anticipated sensory event. Psychological Review, 1959. 66, 203-218.

Creelman. C. D. Human discrimination of auditory duration. Journal of the Acoustical Society of America, 1962, 34, $582-593$.
McGill, W. J, Random fluctuations of response rate. Psychometrika, 1962, 27, 3-17.

Michon. J. A. Timing in temporal tracking. Soesterberg, The Netherlands: Institute for Perception RVO-TNO, 1967.

Treisman, M. Temporal discrimination and the indifference interval: Implications for a model of the "internal clock." Psychological Monographs, 1963, 77, No. 13.

(Received for publication September 13, 1972; revision received January $22,1973$. ) 